\title{
The Hopf algebra structure of renormalizable quantum field theory
}

\author{
Dirk Kreimer \\ Institut des Hautes Etudes Scientifiques \\ 35 rte. de Chartres \\ 91440 Bures-sur-Yvette \\ France \\ E-mail: kreimer@ihes.fr
}

February 2005

\section{Overview}

Renormalization theory is a venerable subject put to daily use in many branches of physics. Here, we focus on its applications in quantum field theory, where a standard perturbative approach is provided through an expansion in Feynman diagrams. Whilst the combinatorics of the Bogoliubov recursion, solved by suitable forest formulas, has been known for a long time, the subject regained interest on the conceptual side with the discovery of an underlying Hopf algebra structure behind these recursions.

Perturbative expansions in quantum field theory are organized in terms of one particle irreducible (1PI) Feynman graphs. The goal is to calculate the corresponding one-particle irreducible Green functions order by order in the coupling constants of the theory, by applying Feynman rules to these 1PI graphs of a renormalizable theory under consideration. This allows to disentangle the problem into an algebraic part and an analytic part.

For the former one studies Feynman graphs as combinatorial objects which lead to the Lie and Hopf algebras discussed below. Feynman rules then assign analytic expressions to these graphs, with the analytic structure of finite renormalized quantum field theory largely dictated by the underlying algebra.

The objects of interest in quantum field theory are the 1PI Green functions. They are parameterized by the quantum numbers, -masses, momenta, spin and such-, of the particles participating in the scattering process under consideration. We call a set of such quantum numbers an external leg structure $\underline{r}$. For 
example, the three terms in the Lagrangian of massless quantum electrodynamics correspond to

$$
\underline{r} \in\{\longrightarrow, \underline{\xi}, \operatorname{mr}\} \text {. }
$$

Note that the Lagrangian $L$ of massless quantum electrodynamics is obtained accordingly as

$$
L=\hat{\phi}(\longrightarrow)^{-1}+\hat{\phi}(\stackrel{\xi}{\underline{\xi}})+\hat{\phi}\left(\mathrm{m} \mathbf{) ^ { - 1 }}=\bar{\psi} \not \partial \psi+\bar{\psi} A \psi+\frac{1}{4} F^{2},\right.
$$

where $\hat{\phi}$ are coordinate space Feynman rules.

The renormalized 1PI Green function in momentum space, $G_{\mathrm{R}}^{r}(\{g\} ;\{p\},\{m\} ; \mu)$, is obtained as the image under renormalized Feynman rules $\phi_{\mathrm{R}}$ applied to a series of graphs

$$
\Gamma^{\underline{r}}=1+\sum_{k=1}^{\infty} g^{k} c_{k}^{r} \equiv 1+\sum_{\operatorname{res}(\Gamma)=\underline{r}} g^{|\Gamma|} \frac{\Gamma}{\operatorname{Sym}(\Gamma)} .
$$

Here $\underline{r}$ is a given such external leg structure, while $c \frac{r}{k}$ is the finite sum of $1 \mathrm{PI}$ graphs having $k$ loops,

$$
c_{k}^{r}=\sum_{\substack{\operatorname{res}(\Gamma)=\underline{x} \\|\Gamma|=k}} \frac{\Gamma}{\operatorname{Sym}(\Gamma)}
$$

and $0<g<1$ is a coupling constant. The generalization to the case of several couplings $\{g\}$ and masses $\{m\}$ is straightforward. In the above, the sum is over all 1PI graphs with the same given external leg structure. We have denoted the map which assigns $\underline{r}$ to a given graph a residue, for example

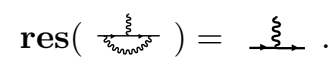

The unrenormalized but regularized Feynman rules $\phi$ assign to a graph a function $\left(\Gamma^{[0]}\right.$ and $\Gamma^{[1] \text { int }}$ being the set of vertices $v$ and internal edges $e$ of $\left.\Gamma\right)$

$$
\phi(\Gamma)(\{p\},\{m\} ; \mu, z)=\int \prod_{v \in \Gamma^{[0]}} \delta^{(4)}\left(\sum_{f \text { incident } v} k_{f}\right) \prod_{e \in \Gamma_{\text {int }}^{[1]}} \operatorname{Prop}\left(k_{e}\right) \frac{d^{4} k_{e}}{4 \pi^{2}},
$$

and formally the unrenormalized Green function

$$
G_{\overline{\mathrm{u}}}^{r}(\{g\} ;\{p\},\{m\} ; \mu, z)=\phi\left(\Gamma^{\underline{r}}\right)(\{g\} ;\{p\},\{m\} ; \mu, z),
$$

which is a function of a suitably chosen regulator $z$. Note that in (6) the fourdimensional Dirac $\delta$ distribution guarantees momentum conservation at each vertex and restricts the number of four-dimensional integrations to the number of independent cycles in the graph. We assume that the reader is familiar with the readily established fact that these integrals suffer from UV singularities 
which render the integration over the momenta in internal cycles ill-defined. We also remind the reader that the problem persists in coordinate space, where one confronts the continuation of products of distributions to regions of coinciding support. We restrict ourselves here to a discussion of the situation in momentum space and refer the reader to the literature for the situation in coordinate space.

Ignoring problems of convergence in the sum over all graphs, the problem of renormalization is to make sense of these functions term by term: We have to determine invertible series $Z^{\underline{r}}(\{g\}, z)$ in the couplings $g$ such that the modified Lagrangian

$$
\tilde{L}=\sum_{\underline{r}} Z^{\underline{r}}(\{g\}, z) \hat{\phi}(\underline{r})
$$

produces a perturbation series in graphs which allows for the removal of the regulator $z$.

This amounts to a transition from unrenormalized to renormalized Feynman rules $\phi \rightarrow \phi_{\mathrm{R}}$. Let us first describe how this transition is achieved using the Lieand Hopf algebra structure of the perturbative expansion which we describe in detail below:

- Decide on the free fields and local interactions of the theory, appropriately specifying quantum numbers (spin, mass, flavor, color and such) of fields, restricting interactions so as to obtain a renormalizable theory.

- Consider the set of all 1PI graphs with edges corresponding to free-field propagators. Define vertices for local interactions. This allows to construct a pre-Lie algebra of graph insertions. Anti-symmetrize this pre-Lie product to get a Lie algebra $\mathcal{L}$ of graph insertions and define the Hopf algebra $\mathcal{H}$ which is dual to the enveloping algebra $\mathcal{U}(\mathcal{L})$ of this Lie algebra.

- Realize that the coproduct and antipode of this Hopf algebra give rise to the forest formula which generates local counterterms upon introducing a Rota-Baxter map, a renormalization scheme in physicists' parlance.

- Use the Hochschild cohomology of this Hopf algebra to show that you can absorb singularities in local counterterms.

- Determine the co-representations of this Hopf algebra to identify the subHopf algebras corresponding to time-ordered products in physical fields. This is most easily achieved by rewriting the Dyson-Schwinger equations using Hochschild one-cocycles.

The last point exhibits close connections in particular between the structure of gauge theories and the co-representation theory of their perturbative Hopf algebras which we discuss below briefly.

This program can be carried out in coordinate space as well as momentum space renormalization. It has given a firm mathematical background to the process 
of renormalization justifying the practice of quantum field theory. The notion of locality has achieved a precise formulation in terms of the Hochschild cohomology of the perturbation expansion. In momentum space, this approach emphasizes the connections to number theory which emerge when one investigates the role of the Hopf algebra primitives which furnish the Hochschild one-cocycles underlying locality.

The next sections describe the above set-up in some detail.

\section{Lie- and Hopf algebras of graphs}

All algebras are supposed to be over some field $\mathbb{K}$ of characteristic zero, associative and unital, and similarly for co-algebras. The unit (and by abuse of notation also the unit map) will be denoted by $\mathbb{I}$, the co-unit map by $\bar{e}$. All algebra homomorphisms are supposed to be unital. A bialgebra $\left(A=\bigoplus_{i=0}^{\infty} A_{i}, m, \mathbb{I}, \Delta, \bar{e}\right)$ is called graded connected if $A_{i} A_{j} \subset A_{i+j}$ and $\Delta\left(A_{i}\right) \subset \bigoplus_{j+k=i} A_{j} \otimes A_{k}$, and if $\Delta(\mathbb{I})=\mathbb{I} \otimes \mathbb{I}$ and $A_{0}=k \mathbb{I}, \bar{e}(\mathbb{I})=1 \in \mathbb{K}$ and $\bar{e}=0$ on $\bigoplus_{i=1}^{\infty} A_{i}$. We call ker $\bar{e}$ the augmentation ideal of $A$ and denote by $P$ the projection $A \rightarrow \operatorname{ker} \bar{e}$ onto the augmentation ideal, $P=i d-\mathbb{I} \bar{e}$. Furthermore, we use Sweedler's notation $\Delta(h)=\sum h^{\prime} \otimes h^{\prime \prime}$ for the coproduct. We define

$$
\operatorname{Aug}^{(k)}=(\underbrace{P \otimes \cdots \otimes P}_{k \text { times }}) \Delta^{k-1}, A \rightarrow\{\operatorname{ker} \bar{e}\}^{\otimes k}
$$

as a map into the $k$-fold tensorproduct of the augmentation ideal. We let $A^{(k)}=$ $\operatorname{ker} \operatorname{Aug}^{(k+1)} / \operatorname{ker} \operatorname{Aug}^{(k)}, \forall k \geq 1$. All bialgebras considered here are bigraded in the sense that

$$
A=\bigoplus_{i=0}^{\infty} A_{i}=\bigoplus_{k=0}^{\infty} A^{(k)},
$$

where $A_{k} \subset \oplus_{j=1}^{k} A^{(j)}$ for all $k \geq 1 . A_{0} \simeq A^{(0)} \simeq \mathbb{K}$.

The first construction we have to study is the pre-Lie algebra structure of 1PI graphs.

\subsection{The Pre-Lie Structure}

For each Feynman graph we have vertices as well as internal and external edges. External edges are edges which have an open end not connected to a vertex. They indicate the particles participating in the scattering amplitude under consideration and each such edge carries the quantum numbers of the corresponding free field. The internal edges and vertices form a graph in their own right. For an internal edge, both ends of the edge are connected to a vertex. 
We are considering 1PI Feynman graphs. A graph $\Gamma$ is $1 \mathrm{PI}$ if and only if all graphs, obtained by removal of any one of its internal edges, are still connected. Such 1PI graphs are naturally graded by their number of independent loops, the rank of their first homology group $H_{[1]}(\Gamma, \mathbb{Z})$. We write $|\Gamma|$ for this degree of a graph $\Gamma$. Note that $|\operatorname{res}(\Gamma)|=0$, where we let $\operatorname{res}(\Gamma)$ be the graph obtained when all edges in $\Gamma_{\text {int }}^{[1]}$ shrink to a point, as before. Note that the graph we obtain in this manner consists of a single vertex, to which the edges $\Gamma_{\text {ext }}^{[1]}$ are attached.

For a 1PI graph $\Gamma$ we write $\Gamma^{[0]}$ for its set of vertices and $\Gamma^{[1]}=\Gamma_{\text {int }}^{[1]} \cup \Gamma_{\text {ext }}^{[1]}$ for its set of internal and external edges. We let $\omega_{\underline{r}}$ be the number of spacetime derivatives appearing in the corresponding monomial in the Lagrangian.

Having specified free quantum fields and local interaction terms between them, one immediately obtains the set of 1PI graphs, and can consider for a given external leg structure $\underline{r}$ the set of graphs with that external leg structure. For a renormalizable theory, we can define a superficial degree of divergence

$$
\omega=\sum_{\underline{r} \in \Gamma_{\text {int }}^{[1]} \cup \Gamma^{[0]}} \omega_{\underline{r}}-4\left|H_{[1]}(\Gamma, \mathbb{Z})\right|,
$$

for each such external leg structure: $\omega(\Gamma)=\omega\left(\Gamma^{\prime}\right)$ if $\operatorname{res}(\Gamma)=\operatorname{res}\left(\Gamma^{\prime}\right)$, all graphs with the same external leg structure have the same superficial degree of divergence, and only for a finite number of distinct external leg structures $\underline{r}$ will this degree indeed signify a divergence.

This leaves a finite number of external leg structures to be considered to which we restrict from now. Our first observation is that there is a natural pre-Lie algebra structure on 1PI graphs.

To this end, we define a bilinear operation

$$
\Gamma_{1} * \Gamma_{2}=\sum_{\Gamma} n\left(\Gamma_{1}, \Gamma_{2} ; \Gamma\right) \Gamma
$$

where the sum is over all 1PI graphs $\Gamma$. Here, $n\left(\Gamma_{1}, \Gamma_{2} ; \Gamma\right)$ is a section coefficient which counts the number of ways a subgraph $\Gamma_{2}$ can be reduced to a point in $\Gamma$ such that $\Gamma_{1}$ is obtained. The above sum is evidently finite as long as $\Gamma_{1}$ and $\Gamma_{2}$ are finite graphs, and the graphs which contribute necessarily fulfill $|\Gamma|=\left|\Gamma_{1}\right|+\left|\Gamma_{2}\right|$ and $\operatorname{res}(\Gamma)=\operatorname{res}\left(\Gamma_{1}\right)$.

One then has:

Theorem 1 The operation $*$ is pre-Lie:

$$
\left[\Gamma_{1} * \Gamma_{2}\right] * \Gamma_{3}-\Gamma_{1} *\left[\Gamma_{2} * \Gamma_{3}\right]=\left[\Gamma_{1} * \Gamma_{3}\right] * \Gamma_{2}-\Gamma_{1} *\left[\Gamma_{3} * \Gamma_{2}\right] .
$$

which is evident when one rewrites the $*$ product in suitable gluing operations. 
To understand this theorem, note that the equation claims that the lack of associativity in the bilinear operation $*$ is invariant under permutation of the elements indexed 2,3. This suffices to show that the anti-symmetrization of this map fulfils a Jacobi identity. Hence we get a Lie algebra $\mathcal{L}$ by anti-symmetrizing this operation:

$$
\left[\Gamma_{1}, \Gamma_{2}\right]=\Gamma_{1} * \Gamma_{2}-\Gamma_{2} * \Gamma_{1} .
$$

This Lie algebra is graded and of finite dimension in each degree. Let us look at a couple of examples for pre-Lie products:

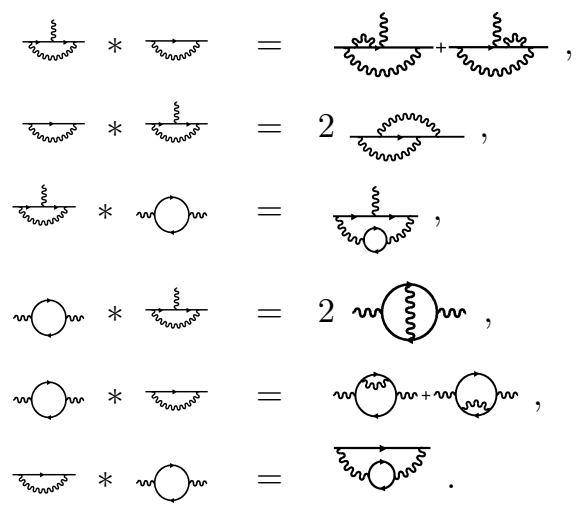

Together with $\mathcal{L}$ one is led to consider the dual of its universal enveloping algebra $\mathcal{U}(\mathcal{L})$ using the theorem of Milnor and Moore. For this we use the above grading by the loop number.

This universal enveloping algebra $\mathcal{U}(\mathcal{L})$ is build from the tensor algebra

$$
\mathbf{T}=\bigoplus_{k} T^{k}, T^{k}=\underbrace{\mathcal{L} \otimes \cdots \otimes \mathcal{L}}_{k \text { times }},
$$

by dividing out the ideal generated by the relations

$$
a \otimes b-b \otimes a=[a, b] \in \mathcal{L} .
$$

Note that in $\mathcal{U}(\mathcal{L})$ we have a natural concatenation product $m_{*}$. Even more, $\mathcal{U}(\mathcal{L})$ carries a natural Hopf algebra structure with this product. For that, the Lie algebra $\mathcal{L}$ furnishes the primitive elements:

$$
\Delta_{*}(a)=a \otimes 1+1 \otimes a, \forall a \in \mathcal{L} .
$$

It is by construction a connected finitely graded Hopf algebra which is cocommutative but not commutative.

We can then consider its graded dual which will be a Hopf algebra $\mathcal{H}(m, \mathbb{I}, \Delta, \bar{e})$ which is commutative but not co-commutative. One finds it upon using a Kronecker pairing

$$
<Z_{\Gamma}, \delta_{\Gamma^{\prime}}>=\left\{\begin{array}{c}
1, \Gamma=\Gamma^{\prime} \\
0, \text { else }
\end{array} .\right.
$$


The space of primitives of $\mathcal{U}(\mathcal{L})$ is in one-to-one correspondence with the set $\operatorname{Indec}(\mathcal{H})$ of indecomposables of $\mathcal{H}$, which is the linear span of its generators.

One finds

\section{Theorem 2}

$$
<Z_{\Gamma_{1}} \otimes Z_{\Gamma_{2}}-Z_{\Gamma_{2}} \otimes Z_{\Gamma_{1}}, \delta_{\Gamma}>=<Z_{\left[\Gamma_{2}, \Gamma_{1}\right]}, \delta_{\Gamma}>
$$

For example, one finds

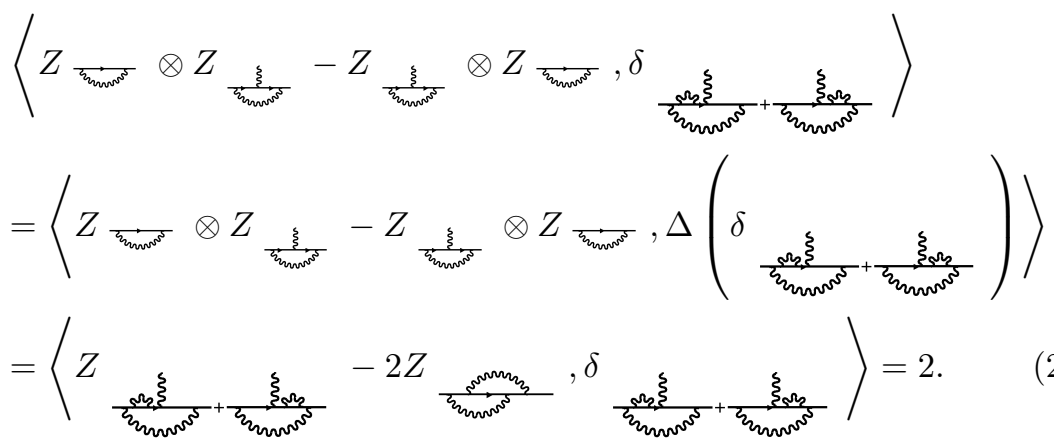

$\mathcal{H}$ is a graded commutative Hopf algebra which suffices to describe renormalization theory as we see in the next section. We have formulated it for the superficially divergent 1PI graphs of the theory with the understanding that the residues of these graphs are in one-to-one correspondence with the terms in the Lagrangian of a given theory. Often it is the case that several terms in a Lagrangian correspond to graphs with the same number and type of external legs, but correspond to different form-factor projections of the graph. In such cases, the above approach can be easily adopted considering suitably colored or labeled graphs. A similar remark applies if one desires to incorporate renormalization of superficially convergent Green functions, which requires nothing more than the consideration of an easily obtained semi-direct product of the Lie algebra of superficially divergent graphs with the abelian Lie algebra of superficially convergent graphs.

\subsection{The principle of multiplicative subtraction}

The above algebra structures are available once one has decided on the set of 1PI graphs of interest. We now use them towards the renormalization of any such chosen local quantum field theory.

From the above, one-particle irreducible graphs $\Gamma$ provide the linear generators $\delta_{\Gamma}$ of the Hopf algebra $\mathcal{H}=\oplus_{i=0}^{\infty} H_{i}$, where $\mathcal{H}_{\text {lin }}=\operatorname{span}\left(\delta_{\Gamma}\right)$ and their disjoint union providing the commutative product. 
Let now $\Gamma$ be a 1 PI graph. We find the Hopf algebra $\mathcal{H}$ as described above to have a co-product explicitely given as $\Delta: \mathcal{H} \rightarrow \mathcal{H} \otimes \mathcal{H}$ :

$$
\Delta(\Gamma)=\Gamma \otimes 1+1 \otimes \Gamma+\sum_{\gamma \subset \Gamma} \gamma \otimes \Gamma / \gamma,
$$

where the sum is over all unions of one-particle irreducible (1PI) superficially divergent proper subgraphs, and we extend this definition to products of graphs so that we get a bi-algebra.

While the Lie bracket inserted graphs into each other, the co-product disentangles them. It is this latter operation which is needed in renormalization theory: we have to render each subgraph finite before we can construct a local counterterm. That is precisely what the Hopf algebra structure maps will do for us.

Having a co-product, two further structure maps of $\mathcal{H}$ are immediate, the counit and the antipode. The co-unit $\bar{e}$ vanishes on any non-trivial Hopf algebra element, $\bar{e}(1)=1, \bar{e}(X)=0$. The antipode is

$$
S(\Gamma)=-\Gamma-\sum_{\gamma \subset \Gamma} S(\gamma) \Gamma / \gamma
$$

We can work out a few co-products and antipodes as follows:

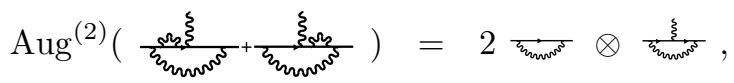

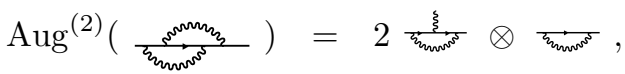

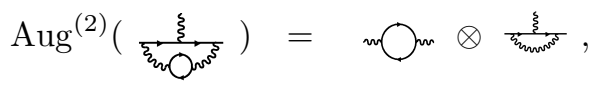

$$
\begin{aligned}
& \operatorname{Aug}^{(2)}(w(\xi) w)=2 \frac{\xi}{\xi m} \otimes m,
\end{aligned}
$$

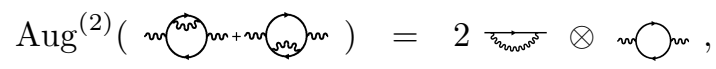

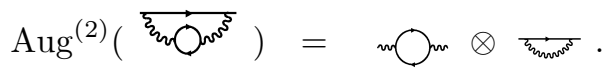

We give just one example for an antipode:

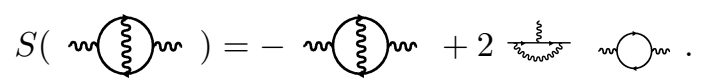

Note that for each term in the sum $\widetilde{\Delta}(\Gamma)=\sum_{i} \Gamma_{(i)}^{\prime} \otimes \Gamma_{(i)}^{\prime \prime}$ we have unique gluing data $G_{i}$ such that

$$
\Gamma=\Gamma_{(i)}^{\prime \prime} \leftarrow_{G_{i}} \Gamma_{(i)}^{\prime}, \forall i .
$$

These gluing date describe the necessary bijections to glue the components $\Gamma_{(i)}^{\prime}$ back into $\Gamma_{(i)}^{\prime \prime}$ so as to obtain $\Gamma$ : using them, we can reassemble the whole 
from its parts. Each possible gluing can be interpreted as a composition in the insertion operad of Feynman graphs.

We have by now obtained a Hopf algebra generated by combinatorial elements, 1PI Feynman graphs. Its existence is automatic from the above choices of interactions and free fields. What is left to us is a structural analysis of these algebras for the renormalizable theories we are confronted with in four spacetime dimensions.

The assertion underlying perturbation theory is the fact that meaningful approximations to physical observable quantities can be found by evaluating these graphs using Feynman rules.

First, as disjoint scattering processes give rise to independent amplitudes one is led to the study of characters of the Hopf algebra, maps $\phi: \mathcal{H} \rightarrow V$ such that $\phi \circ m=m_{V}(\phi \otimes \phi)$.

Such maps assign to any element in the Hopf algebra an element in a suitable target space $V$. The study of tree-level amplitudes in lowest order perturbation theory justifies to assign to each edge a propagator and to each elementary scattering process a vertex which define the Feynman rules $\phi(\operatorname{res}(\Gamma))$ and the underlying Lagrangian, on the level of residues of these very graphs. As graphs themselves are constructed from edges and vertices, such residues, one is led to assign to each Feynman graph an evaluation in terms of an integral over the continues quantum numbers assigned to edges or vertices, which leads to the familiar integrals over momenta in closed loops mentioned before.

Then, with the Feynman rules providing a canonical character $\phi$, we will have to make one further choice: a renormalization scheme. The need for such a choice is no surprise: after all we are eliminating short-distance singularities in the graphs which renders their remaining finite part ambiguous, albeit in a most interesting manner.

Hence we choose a map $R: V \rightarrow V$, from which we obviously demand that is does not modify the UV-singular structure, and furthermore that it obeys

$$
R(x y)+R(x) R(y)=R(R(x) y)+R(x R(y)),
$$

an equation which guarantees the multiplicativity of renormalization and is at the heart of the Birkhoff decomposition which emerges below: it tells us that elements in $V$ split into two parallel subalgebras given by the image and kernel of $R$. Algebras for which such a map exists are known as Rota-Baxter algebras. Rota-Baxter algebras play a role for associative algebras which is similar to the role Yang-Baxter algebras play for Lie algebras. The structure of these algebras allows to connect renormalization theory to integrable systems. Also, most of the results obtained initially for a specific renormalization scheme like minimal subtraction can be obtained in general upon a structural analysis of the corresponding Rota-Baxter algebras.

To see how all the above comes together in renormalization theory we define a 
further character $S_{R}^{\phi}$ which deforms $\phi \circ S$ slightly and delivers the counterterm for $\Gamma$ in the renormalization scheme $R$ :

$$
S_{R}^{\phi}(\Gamma)=-R m_{V}\left(S_{R}^{\phi} \otimes \phi \circ P\right) \Delta=-R[\phi(\Gamma)]-R\left[\sum_{\gamma \subset \Gamma} S_{R}^{\phi}(\gamma) \phi(\Gamma / \gamma)\right]
$$

which should be compared with the undeformed

$$
\phi \circ S=m_{V}(S \circ \phi \otimes \phi \circ P) \Delta=-\phi(\Gamma)-\sum_{\gamma \subset \Gamma} \phi \circ S(\gamma) \phi(\Gamma / \gamma) .
$$

The fact that $R$ is a Rota-Baxter map ensures that $S_{R}^{\phi}$ is an element of the character group $\mathcal{G}$ of the Hopf algebra, $S_{R}^{\phi} \in \operatorname{Spec}(\mathcal{G})$. Note that we now have determined the modified lagrangian:

$$
Z^{\underline{r}}=S_{R}^{\phi}\left(\Gamma^{\underline{r}}\right)
$$

The classical results of renormalization theory follow immediately using this group structure: We obtain the renormalization of $\Gamma$ by the application of a renormalized character

$$
S_{R}^{\phi} \star \phi(\Gamma)=m_{v}\left(S_{R}^{\phi} \otimes \phi\right) \Delta
$$

and Bogoliubov's $\bar{R}$ operation as

$$
\bar{R}(\Gamma)=m_{V}\left(S_{R}^{\phi} \otimes \phi\right)(\operatorname{id} \otimes P) \Delta(\Gamma)=\phi(\Gamma)+\sum_{\gamma \subset \Gamma} S_{R}^{\phi}(\gamma) \phi(\Gamma / \gamma),
$$

so that we have

$$
S_{R}^{\phi} \star \phi(\Gamma)=\bar{R}(\Gamma)+S_{R}^{\phi}(\Gamma)
$$

Here, $S_{R}^{\phi} \star \phi$ is an element in the group of characters of the Hopf algebra, with the group law given by the convolution

$$
\phi_{1} \star \phi_{2}=m_{V} \circ\left(\phi_{1} \otimes \phi_{2}\right) \circ \Delta
$$

so that the co-product, co-unit and co-inverse (the antipode) give the product, unit and inverse of this group, as befits a Hopf algebra. This Lie group has the previous Lie algebra $\mathcal{L}$ of graph insertions as its Lie algebra: $\mathcal{L}$ exponentiates to $\mathcal{G}$.

What we have achieved at this moment is a local renormalization of quantum field theory. Let $M^{\underline{r}}$ be a monomial in the lagrangian $L$ of degree $\omega_{\underline{r}}$,

$$
M^{\underline{r}}=D_{\underline{r}}\{\phi\} .
$$

Then one can prove using the Hochschild cohomology of $\mathcal{H}$ : 
Theorem 3 (locality)

$$
Z^{\underline{r}} D_{\underline{r}}\{\phi\}=D_{\underline{r}} Z^{\underline{r}}\{\phi\},
$$

renormalization commutes with infinitesimal space-time variations of the fields.

We can now work out the renormalization of a Feynman graph $\Gamma$.

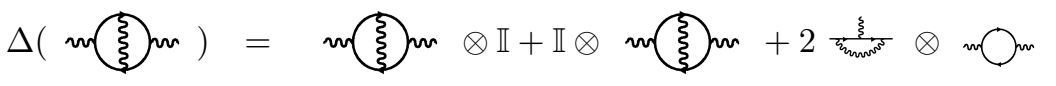

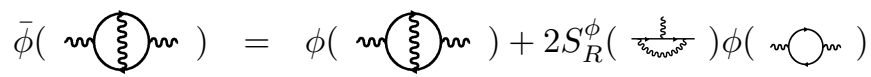

$$
\begin{aligned}
& =\phi(v(\xi) v r)-2 R\left[\phi\left(\frac{\xi}{\xi_{\text {mans }}}\right)\right] \phi(w \mathrm{Or})
\end{aligned}
$$

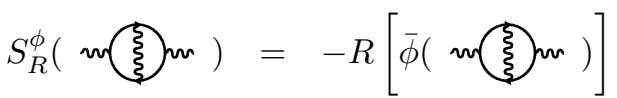

$$
\begin{aligned}
& \phi_{R}(v(\xi) \sim r) \equiv S_{R}^{\phi} \star \phi(v(\xi) \sim r)=[\mathrm{id}-R] \circ[\bar{\phi}(v(\xi) \sim r)] \text {. }
\end{aligned}
$$

In the above, we have given all formulas in their recursive form. Zimmermann's original forest formula solving this recursion is obtained when we trace our considerations back to the fact that the co-product can be written in non-recursive form as a sum over forests, and similarly for the antipode.

\section{Diffeomorphisms of physical parameters}

In the above, we effectively obtained a Birkhoff decomposition of the Feynman rules $\phi \in \operatorname{Spec}(\mathcal{G})$ into two characters $\phi_{+}^{R}=S_{R}^{\phi} \star \phi \in \operatorname{Spec}(\mathcal{G})$ and $\phi_{-}^{R}=S_{R}^{\phi} \in$ $\operatorname{Spec}(\mathcal{G})$, for any Rota-Baxter map $R$. Thanks to Atkinson's theorem this is possible for any renormalization scheme $R$. For the minimal subtraction scheme it amounts to the decomposition of the Laurent series $\phi(\Gamma)(\epsilon)$, which has poles of finite order in the regulator $\epsilon$, into a part holomorphic at the origin and a part holomorphic at complex infinity. This has a particularly nice geometric interpretation upon considering the Birkhoff decomposition of a loop around the origin, providing the clutching data for the two half-spheres defined by that very loop.

Whilst in this manner a satisfying understanding of perturbative renormalization is obtained, alas the character group $\mathcal{G}$ is a rather big and poorly understood object. On the other hand, renormalization can be captured by the study of diffeomorphisms of physical parameters, as by the very definition the range of allowed modification in renormalization theory is the variation of the coefficients of monomials $\hat{\phi}(\underline{r})$ of the underlying Lagrangian

$$
L=\sum_{\underline{r}} Z^{\underline{r}} \hat{\phi}(\underline{r})
$$


Thus one desires to obtain the whole Birkhoff decomposition at the level of diffeomorphisms of the coupling constants.

The crucial step toward that goal is to realize the role of a standard quantum field-theoretic formula of the form

$$
g_{\text {new }}=g_{\text {old }} Z^{g},
$$

where

$$
Z^{g}=\frac{Z^{v}}{\prod_{e \in \operatorname{res}(v)_{\text {ext }}^{[1]}} \sqrt{Z^{e}}}
$$

for some vertex $v$, which obtains the new coupling in terms of a diffeomorphism of the old. This formula provides indeed a Hopf algebra homomorphism from the Hopf algebra of diffeomorphisms to the Hopf algebra of Feynman graphs, regarding $Z^{g}$, a series over counterterms for all 1PI graphs with the external leg structure corresponding to the coupling $g$, in two different ways: it is at the same time a formal diffeomorphism in the coupling constant $g_{\text {old }}$ and a formal series in Feynman graphs. As a consequence, there are two competing coproducts acting on $Z_{g}$. That both give the same result defines the required homomorphism, which transposes to a homomorphism from the largely unknown group of characters of $\mathcal{H}$ to the one-dimensional diffeomorphisms of this coupling.

In summary, one finds that a couple of basic facts enable one to the transition from the foreign territory of the abstract group of characters of a Hopf algebra of Feynman graphs (which, by the way, equals the Lie group assigned to the Lie algebra with universal enveloping algebra the dual of this Hopf algebra) to the rather concrete group of diffeomorphisms of physical observables. These steps are:

- Recognize that $Z$ factors are given as counterterms over formal series of graphs starting with 1 , graded by powers of the coupling, hence invertible.

- Recognize the series $Z_{g}$ as a formal diffeomorphism, with Hopf algebra coefficients.

- Establish that the two competing Hopf algebra structures of diffeomorphisms and graphs are consistent in the sense of a Hopf algebra homomorphism.

- Show that this homomorphism transposes to a Lie algebra and hence Lie group homomorphism.

The effective coupling $g_{\text {eff }}(\varepsilon)$ now allows for a Birkhoff decomposition in the space of formal diffeomorphisms:

Theorem 4 Let the unrenormalized effective coupling constant $g_{\mathrm{eff}}(\varepsilon)$ viewed as a formal power series in $g$ be considered as a loop of formal diffeomorphisms 
and let $g_{\mathrm{eff}}(\varepsilon)=\left(g_{\mathrm{eff}_{-}}\right)^{-1}(\varepsilon) g_{\mathrm{eff}_{+}}(\varepsilon)$ be its Birkhoff decomposition in the group of formal diffeomorphisms. Then the loop $g_{\mathrm{eff}_{-}}(\varepsilon)$ is the bare coupling constant and $g_{\mathrm{eff}_{+}}(0)$ is the renormalized effective coupling.

The above results hold as they stand for any massless theory which provides a single coupling constant. If there are multiple interaction terms in the Lagrangian, one finds similar results relating the group of characters of the corresponding Hopf algebra to the group of formal diffeomorphisms in the multidimensional space of coupling constants.

\section{The role of Hochschild cohomology}

The Hochschild cohomology of the combinatorial Hopf algebras which we discuss here plays three major roles in quantum field theory: it allows to prove locality from the accompanying filtration by the augmentation degree coming from the kernels $\operatorname{ker}^{\operatorname{Aug}^{(k)}}$, it allows to write the quantum equations of motion in terms of the Hopf algebra primitives, elements in $\mathcal{H}_{\text {lin }} \cap\left\{\operatorname{ker~Aug}^{(2)} / \operatorname{ker~Aug}^{(1)}\right\}$, and identifies the relevant sub-Hopf algebras formed by time-ordered products. Before we discuss these properties, let us first introduce the relevant Hochschild cohomology.

\subsection{Hochschild cohomology of bialgebras}

Let $(A, m, \mathbb{I}, \Delta, \epsilon)$ be a bialgebra, as before. We regard linear maps $L: A \rightarrow A^{\otimes n}$ as $n$-cochains and define a coboundary map $b, b^{2}=0$, by

$$
b L:=(\mathrm{id} \otimes L) \circ \Delta+\sum_{i=1}^{n}(-1)^{i} \Delta_{i} \circ L+(-1)^{n+1} L \otimes \mathbb{I}
$$

where $\Delta_{i}$ denotes the coproduct applied to the $i$-th factor in $A^{\otimes n}$, which defines the Hochschild cohomology of $A$.

For the case $n=1,(55)$ reduces to, for $L: A \rightarrow A$,

$$
b L=(\mathrm{id} \otimes L) \circ \Delta-\Delta \circ L+L \otimes \mathbb{I} .
$$

The category of objects $(A, C)$ which consists of a commutative bialgebra $A$ and a Hochschild one-cocycle $C$ on $A$ has an initial object $\left(\mathcal{H}_{\mathrm{rt}}, B_{+}\right)$, where $\mathcal{H}_{\mathrm{rt}}$ is the Hopf algebra of (non-planar) rooted trees and the closed but non-exact onecocycle $B_{+}$grafts a product of rooted trees together at a new root as described below.

The higher $(n>1)$ Hochschild cohomology of $\mathcal{H}_{\mathrm{rt}}$ vanishes, but the closedness of $B_{+}$will turn out to be crucial for what follows. 


\subsection{The Hopf algebra of rooted trees}

A rooted tree is a simply-connected contractible compact graph with a distinguished vertex, the root. A forest is a disjoint union of rooted trees. Isomorphisms of rooted trees or forests are isomorphisms of graphs preserving the distinguished vertex/vertices. Let $t$ be a rooted tree with root $o$. The choice of $o$ determines an orientation of the edges of $t$, away from the root, say. Forests are graded by the numbers of vertices they contain.

Let $\mathcal{H}_{\text {rt }}$ be the free commutative algebra generated by rooted trees. The commutative product in $\mathcal{H}_{\mathrm{rt}}$ corresponds to the disjoint union of trees, such that monomials in $\mathcal{H}_{\mathrm{rt}}$ are scalar multiples of forests. We demand that the linear operator $B_{+}$on $\mathcal{H}_{\text {rt }}$, defined by

$$
\begin{aligned}
B_{+}(\mathbb{I}) & =\bullet \\
B_{+}\left(t_{1} \ldots t_{n}\right) & =\bullet_{t_{1} \ldots t_{n}},
\end{aligned}
$$

is a Hochschild 1-cocycle, which makes $\mathcal{H}_{\mathrm{rt}}$ a Hopf algebra. The resulting coproduct can be described as follows

$$
\Delta(t)=\mathbb{I} \otimes t+t \otimes \mathbb{I}+\sum_{a d m . c} P_{c}(t) \otimes R_{c}(t),
$$

where the sum goes over all admissible cuts of the tree $t$. Such a cut of $t$ is a nonempty set of edges of $t$ to be removed. The forests which are disconnected from the root upon removal of those edges is denoted by $P_{c}(t)$ and the part which remains connected to the root is denoted by $R_{c}(t)$. A cut $c(t)$ is admissible if for each vertex $l$ of $t$ it contains at most one edge on the path from $l$ to the root.

This Hopf algebra of non-planar rooted trees is the universal object after which all such commutative Hopf algebras $\mathcal{H}$ providing pairs $(\mathcal{H}, B)$, for $B$ a Hochschild one-cocycle, are formed:

Theorem 5 The pair $\left(\mathcal{H}_{\mathrm{rt}}, B_{+}\right)$, unique up to isomorphism, is universal among all such pairs. In other words for any pair $(\mathcal{H}, B)$ where $\mathcal{H}$ is a commutative Hopf algebra and $B$ a closed non-exact one-cocycle, there exists a unique Hopf algebra morphism $\mathcal{H}_{\mathrm{rt}} \stackrel{\rho}{\rightarrow} \mathcal{H}$ such that $B \circ \rho=\rho \circ B_{+}$.

This theorem suggests to investigate the Hochschild cohomology of the Hopf algebras of 1PI Feynman graphs. It largely illuminates the structure of 1PI Green functions.

\subsection{The roles of Hochschild cohomology}

The Hochschild cohomology of the Hopf algebras of 1PI graphs sheds light on the structure of 1PI Green function in at least four different ways: 
- it gives a coherent proof of locality of counterterms -the very fact that

$$
\left[Z^{\underline{r}}, D_{\underline{r}}\right]=0
$$

the coefficients in the Lagrangian remain independent of momenta, and hence the Lagrangian a polynomial expression in fields and their derivatives;

- the quantum equation of motions take a very succinct form identifying the Dyson kernels with the primitives of the Hopf algebra;

- sub-Hopf algebras emerge from the study of the Hochschild cohomology which connect the representation theory of these Hopf algebras to the structure of theories with internal symmetries;

- these Hopf algebras are intimately connected to the structure of transcendental functions like the generalized polylogarithms which play a prominent role these days ranging from applied particle physics to recent developments in mathematics.

To determine the Hochschild one-cocycles of some Feynman graph Hopf algebra $\mathcal{H}$, one determines first the primitives graphs $\gamma$ of the Hopf algebra, which by definition fulfill

$$
\Delta(\gamma)=\gamma \otimes \mathbb{I}+\mathbb{I} \otimes \gamma
$$

Using the pre-Lie product above, one then determines maps

$$
B_{+}^{\gamma}: \mathcal{H} \rightarrow \mathcal{H}_{\text {lin }}
$$

such that

$$
B_{+}^{\gamma}(h)=B_{+}^{\gamma}(h) \otimes \mathbb{I}+\left(\mathrm{id} \otimes B_{+}^{\gamma}\right) \Delta(h),
$$

where $B_{+}^{\gamma}(h)=\sum_{\Gamma} n(\gamma, h, \Gamma) \Gamma$. The coefficients $n(\gamma, h, \Gamma)$ are closely related to the section coefficients we had before.

Using the definition of the Bogoliubov map $\bar{\phi}$ this immediately shows that

$$
S_{R}^{\phi}\left(B_{+}^{\gamma}(h)\right)=\int \mathrm{D}_{\gamma} \leftarrow G_{i} \phi_{\mathrm{R}}(h),
$$

which proves locality of counterterms upon recognizing that $B_{+}^{\gamma}$ increases the augmentation degree. Here, the insertion of the functions for the subgraph is achieved using the relevant gluing data of (36).

To recover the quantum equation of motions from the Hochschild cohomology, one proves that

$$
\Gamma^{\underline{r}}=1+\sum_{\gamma} \frac{g^{|\gamma|}}{\operatorname{sym}(\gamma)} B_{+}^{\gamma}\left(X_{\gamma}\right)
$$

where

$$
X_{\gamma}=\prod_{e \in \gamma_{\mathrm{int}}^{[1]}} \prod_{v \in \gamma^{[0]}} \Gamma^{\underline{v}} / \Gamma^{\underline{e}}
$$


has the required solution. Upon application of the Feynman rules the maps $B_{+}^{\gamma}$ turn to the integral kernels of the usual Dyson-Schwinger equations. This allows for new non-perturbative approaches which are a current theme of investigation.

Finally, we note that the one-cocycles introduced above allow to determine sub Hopf algebras of the form

$$
\Delta\left(c_{n}^{\frac{r}{n}}\right)=\sum P\left(\left\{c_{j}^{s}\right\}\right) \otimes c_{j}^{r},
$$

where the $c_{j}^{s}$ are defined in Eq.(3). These algebras do not necessitate the considerations of single Feynman graphs any longer, but allow to establish renormalization directly for the sum of all graphs at a given loop order. They hence establish a Hopf algebra structure on time-ordered products in momentum space. For theories with internal symmetries one expects and indeed finds that the existence of these sub-algebras establishes relations between graphs which are the Slavnov-Taylor identities between the couplings in the lagrangian.

\title{
5 Outlook
}

Thanks to the Hopf and Lie algebra structures described above quantum field theory has started to reveal its internal mathematical structure in recent years, which connects it to motivic theory and arithmetic geometry. Conceptually, quantum field theory has been the most sophisticated means by which a physicist can describe the character of the physical law. We slowly start understanding that in its short-distance singularities it encapsulates concepts of matching beauty. We can expect local point-particle quantum field theory to remain a major topic of mathematical physics investigation in the foreseeable future indeed.

\section{See also}

perturbative quantum field theory

Dyson-Schwinger equations

BPHZ renormalization

the renormalization group

\section{Keywords}

\author{
Renormalization \\ Renormalization group \\ Feynman graphs
}


Hopf algebras

\section{References}

[1] D. Kreimer, New mathematical structures in renormalizable quantum field theories, Annals Phys. 303 (2003) 179 [arXiv:hep-th/0211136].

[2] D. Kreimer, The residues of quantum field theory: Numbers we should know, arXiv:hep-th/0404090.

[3] A. Connes and D. Kreimer, Hopf algebras, renormalization and noncommutative geometry, Commun. Math. Phys. 199 (1998) 203 [arXiv:hepth/9808042].

[4] A. Connes and D. Kreimer, Renormalization in quantum field theory and the Riemann-Hilbert problem. II: The beta-function, diffeomorphisms and the renormalization group, Commun. Math. Phys. 216 (2001) 215 [arXiv:hep-th/0003188].

[5] A. Connes and M. Marcolli, From physics to number theory via noncommutative geometry. II: Renormalization, the Riemann-Hilbert correspondence, and motivic Galois theory, arXiv:hep-th/0411114. 